\title{
Large eddy simulation of suppression of turbulent line fires by base-injected water mist
}

\author{
S. Vilfayeau, T. Myers, A.W. Marshall, A. Trouvé* \\ Department of Fire Protection Engineering, University of \\ Maryland, College Park, MD 20742, USA
}

\begin{abstract}
The objective of this study is to bring basic information on the mechanisms that control flame cooling effectiveness in water mist fire suppression systems. The study is based on well-resolved large eddy simulations (LES) of buoyancy-driven, turbulent, methane-air diffusion flames exposed to water mist injected through a controlled air co-flow. Simulations are performed using an LES solver called FireFOAM and using a modified version recently enhanced with a new flame extinction model based on the concepts of a critical flame Damköhler number for extinction and a critical gas temperature for re-ignition. The numerical simulations provide global information on the flame response to changes in the water mist load as well as spatially-resolved information on the structure of the flame-based heat release rate processes and mist-based evaporation processes. The results suggest that maximum suppression is obtained when mist droplets are entrained into the flame base region.
\end{abstract}

${ }^{*}$ Corresponding author. Address: University of Maryland, 3104D J.M Patterson Bldg., College Park, MD 20742-3031, USA. Fax:+1 301-405-9383.

Email address: atrouve@umd.edu (A. Trouvé)

Preprint submitted to Proceedings of the Combustion Institute

June 2, 2016

(C) 2016. This manuscript version is made available under the Elsevier user license

http://www.elsevier.com/open-access/userlicense/1.0/ 
Keywords: Flame Extinction, Fire Suppression, Water Mist, Turbulent Buoyant Diffusion Flame, Large Eddy Simulation

1

\section{Introduction}

While water-based fire suppression systems provide an attractive and popular solution to the problem of fire safety in the built environment, a fundamental understanding of the factors that control the performance of these systems is still lacking [1-3]. When evaluating the fire suppression performance of water sprays, it is useful to differentiate between systems that achieve suppression by flame cooling, i.e., systems that reduce the fire intensity by acting directly on combustion (e.g., by increasing the probability of flame extinction), and systems that achieve suppression by fuel cooling, i.e., systems that reduce the fire intensity by acting on fuel formation (e.g., by wetting active fuel surfaces and decreasing the rate of pyrolysis, or by wetting inactive fuel surfaces and inhibiting further flame spread). The ability of a given system to achieve flame cooling or fuel cooling depends on its ability to deliver the liquid water to the location of the flame or to that of the fuel surfaces and is therefore strongly related to the droplet size distribution in the liquid spray. Conventional sprinkler systems produce large droplets (larger than 100 microns) that penetrate the gas flow and evaporate slowly; large droplets have a low probability of acting (i.e., evaporating) at the flame location and a high probability of wetting fuel surfaces; sprinkler systems achieve fire suppression primarily by fuel cooling. In contrast, mist systems produce small droplets (smaller than 100 microns) that move with the gas flow and evaporate quickly; small droplets have a relatively high 
probability of reaching the flame location (at least in confined spaces) and a low probability of wetting fuel surfaces; mist systems achieve fire suppression primarily by flame cooling (note that mists systems have also the ability to block radiation transport via droplet absorption and scattering effects and can therefore also provide some form of fuel cooling).

One of the main objectives of fire suppression research is to fill the knowledge gap between the engineering-level performance indicators that are used in the design of practical water-based fire suppression systems and the research-level performance indicators that are produced by fundamental studies. Engineering-level performance indicators are global parameters that typically compare the evaporative cooling power of a sprinkler or mist system to the size of the fire at activation time: $I_{g l o b a l}=\left(\dot{m}_{w} \Delta H_{v}\right) / \dot{Q}_{c}$, where $\dot{m}_{w}$ is the mass flow rate of delivered water (in units of $\mathrm{kg} / \mathrm{s}$ ), $\Delta H_{v}$ the latent heat of evaporation of water (in J/kg), and $\dot{Q}_{c}$ the combustion power prior to suppression (in W). Fire suppression requires values of $I_{\text {global }}$ that are strongly configuration-dependent and that vary from values of order 1 to values much larger than $1[1-3]$.

Research-level performance indicators are local parameters that take different forms, depending on whether fire suppression is achieved by flame cooling or by fuel cooling. In the case of flame cooling, a typical indicator compares the rate of heat extraction by evaporative cooling (measured at the flame surface) to the rate of energy release by combustion: $I_{\text {flame }}=\left(\dot{\omega}_{w}^{\prime \prime} \Delta H_{v}\right) /\left(\dot{m}_{f, c}^{\prime \prime} \Delta H_{c}\right)$, where $\dot{\omega}_{w}^{\prime \prime}$ is the local mass evaporation rate of liquid water (in $\mathrm{kg} / \mathrm{s} / \mathrm{m}^{2}$, i.e., per unit area of flame surface), $\dot{m}_{f, c}^{\prime \prime}$ the local fuel mass consumption rate by combustion (in $\mathrm{kg} / \mathrm{s} / \mathrm{m}^{2}$, also per unit 
area of flame surface and measured prior to suppression), and $\Delta H_{c}$ the heat of combustion (in $\mathrm{J} / \mathrm{kg}$ ). Successful flame cooling requires values of $I_{\text {flame }}$ of order 0.1 , which typically corresponds to values of the critical water mass flux on the order of a few tens of $\mathrm{g} / \mathrm{s} / \mathrm{m}^{2}[4-7]$. Alternative representative measures of the water loading requirement for flame cooling are a liquid volume fraction on the order of $100 \mathrm{ppm}$ or a liquid mass fraction on the order of $10 \%$ [8].

We consider in the present study the problem of flame cooling by a water mist and focus in particular on the relationship between the spray effectiveness indicators $I_{\text {global }}$ and $I_{\text {flame }}$. The global indicator $I_{\text {global }}$ is not suitable to measure spray effectiveness because only a fraction of the delivered liquid water will actually be transported to the flame and thereby contribute to flame cooling; in other words, $I_{\text {global }}$ incorrectly incorporates water mist that either fails to evaporate or that evaporates at locations that do not impact the flame. A clear illustration of this problem is found in Refs. $[9,10]$ which present a direct numerical simulation study of flame extinction due to water mist in laminar co-flow methane-air and methanol-air diffusion flames. In the most efficient case corresponding to a base injection of the mist inside the air-coflow and at low flow velocities $(25 \mathrm{~cm} / \mathrm{s})$, flame extinction is achieved for different values of $I_{\text {global }}: I_{\text {global }} \approx 0.75$ in the case of 50 microns droplets and $I_{\text {global }} \approx 3$ in the case of 150 microns droplets (in these estimates, we use $\Delta H_{v}=3.7 \mathrm{MJ} / \mathrm{kg}$, a representative value that corresponds to the enthalpy change from liquid water at $293 \mathrm{~K}$ to steam at $1500 \mathrm{~K}$ ). Extinction values of $I_{\text {global }}$ are also found to be much higher in the case of higher mist injection velocities [9]. Thus it is found in Ref. [9] that the value $I_{\text {global }} \approx 0.75$ 
may correspond to a suppressed flame (50 microns droplets case), to a flame that has a much reduced (more than 50\%) combustion intensity (100 microns droplets case) or to a flame that is only weakly affected by the presence of the water mist (150 microns droplets case or cases with high injection velocities). These results demonstrate that the global indicator $I_{\text {global }}$ does not provide an accurate measure of suppression performance.

Our objectives in the present study are to provide additional insight into basic mist-flame interactions and to replace $I_{\text {global }}$ by a more suitable fire suppression performance indicator. The study is based on detailed numerical simulations which provide spatially-resolved information on the distributions of evaporation and combustion rates. We choose to consider a new configuration corresponding to a buoyant, turbulent, methane-fueled, slotburner diffusion flame with a mono-disperse water spray injected through a controlled air co-flow. This configuration is being currently developed at the University of Maryland [11] (note that experimental data with mist are not available yet and that the present study is a standalone numerical investigation). The numerical solver is an advanced large eddy simulation (LES) solver called FireFOAM, developed by FM Global [12, 13] and based on a general-purpose Computational Fluid Dynamics (CFD) solver called OpenFOAM [14]. The FireFOAM solver has been recently enhanced with a new flame extinction model $[15,16]$. The LES solver and numerical configuration are presented in Section 2. Results are presented in Section 3, including results documenting changes in combustion intensity in response to changes in water mist load. Conclusions are drawn in Section 4. 


\section{Numerical solver and configuration}

\subsection{Numerical configuration}

The configuration adopted in the present study is an experimental configuration currently being developed at the University of Maryland [11]. The configuration corresponds to a buoyant, turbulent, methane-fueled, slotburner diffusion flame with a controlled co-flow. Flames are stabilized above a 5-cm-wide and 50-cm-deep Wolfhard-Parker burner surrounded by a small horizontal plate and an air co-flow (Fig. 1). The plate is 15-cm-wide and 60-cm-deep and is made of Kaowool ceramic board. The plate adds an horizontal component to the flow motion near the flame base and was introduced in previous work with the intent to promote faster transition to turbulent flow conditions [11]. The air co-flow is 50-cm-wide and 75-cm-deep. The methane velocity is $6 \mathrm{~cm} / \mathrm{s}$; the methane mass flow rate is $1 \mathrm{~g} / \mathrm{s}$; the total heat release rate of the unsuppressed flame is $50 \mathrm{~kW}$.

In the baseline numerical configuration with mist, the air co-flow is saturated with water vapor $(100 \%$ humidity) and loaded with a mono-disperse liquid water spray. The air co-flow velocity is $29 \mathrm{~cm} / \mathrm{s}$ and the air mass flow rate is $100 \mathrm{~g} / \mathrm{s}$. Selected droplet diameters are $30 \mu \mathrm{m}$ and $60 \mu \mathrm{m}$ (larger droplets are too heavy to guarantee successful entrainment into the flame region, in particular when the flame strength is reduced). The initial droplet velocity is equal to the air co-flow velocity; the mist mass flow rate varies between 0 and $18 \mathrm{~g} / \mathrm{s}$; the mist volume (mass) fraction varies between 0 and $220 \mathrm{ppm}(0$ and $15.3 \%)$.

We also consider below a modified configuration in which the co-flow is split into an inner area corresponding to air (40\% humidity) without water 
mist and an outer area corresponding to air (100\% humidity) with water mist. The no-mist inner co-flow is introduced in order to protect the base of the flame from suppression. The inner area is 25-cm-wide and 70-cm-deep; the air co-flow velocity in the inner area (also referred to as the protective co-flow) varies between 20 and $50 \mathrm{~cm} / \mathrm{s}$ (and thereby provides different levels of protection). The air co-flow velocity in the outer area is $42 \mathrm{~cm} / \mathrm{s}$; the air mass flow rate is $100 \mathrm{~g} / \mathrm{s}$; the mist mass flow rate is $18 \mathrm{~g} / \mathrm{s}$.

\subsection{Solver}

FireFOAM $[12,13]$ is based on OpenFOAM [14], an open-source generalpurpose CFD software package. FireFOAM is an object-oriented, $\mathrm{C}++-$ based, second-order accurate, finite volume solver with implicit time integration; PISO and SIMPLE types of solution procedures are used to couple sequentially-solved equations; the solver features advanced meshing capabilities (the mesh geometry is a structured or unstructured polyhedral mesh); it also features a massively parallel computing capability using Message Passing Interface (MPI) protocols.

FireFOAM uses a Favre-filtered compressible-flow LES formulation and provides a choice between several modeling options for the treatment of turbulence, combustion and thermal radiation. In the present study, subgridscale turbulence is described using the one-equation eddy viscosity model (a model based on solving a transport equation for subgrid-scale turbulent kinetic energy or TKE) [17]. Combustion is described using the classical concept of a global combustion equation combined with the Eddy Dissipation Concept (EDC) model [18]. Thermal radiation is described by solving the radiative transfer equation (RTE). Radiative properties are considered as 
spectrally-averaged quantities and a gray medium is assumed. In FireFOAM, the RTE is solved using a finite volume implementation of the discrete ordinate method [19]. In the present study, the description of thermal radiation is simplified by assuming a non-scattering, non-absorbing, optically-thin medium and using the empirical concept of a user-prescribed global radiative loss fraction $\chi_{\text {rad }}$; we use $\chi_{\text {rad }}=23 \%$ [11]. The assumption of an emitting but non-absorbing medium as well as that of a fixed radiative loss fraction should be viewed as an intermediate modeling step. Also note that the present methane-fueled flames are only weakly sooting and that soot is simply neglected in the model formulation.

For the water spray, FireFOAM uses a classical Lagrangian-based modeling approach that treats the liquid phase as a discrete phase, injects representative (spherical-shaped) droplets according to prescribed size and velocity boundary conditions, tracks the individual trajectories of the representative droplets, and includes two-way coupling between the gas and liquid phases, with a limitation to dilute conditions (this limitation is deemed acceptable for the present application) [20]. We refer the reader to Refs. [4, 21, 22] for a description of the Lagrangian-based droplet equations and Eulerian-based gas equations.

We now turn to a description of the flame extinction model in FireFOAM. The FireFOAM solver has been recently enhanced with a new flame extinction model based on the concepts of a critical flame Damköhler number for extinction and a critical gas temperature for re-ignition $[15,16]$. The model was initially formulated using the concept of mixture fraction [15]; because mixture fraction does not apply to problems with multiple fuels and multi- 
ple oxidizer streams, the model has been recently re-formulated to a more general framework that does not use mixture fraction [23].

The combustion model in FireFOAM uses a classical global combustion equation: $\mathrm{CH}_{4}+2 \mathrm{O}_{2} \rightarrow \mathrm{CO}_{2}+2 \mathrm{H}_{2} \mathrm{O}$ (GR1). Global reaction (GR1) corresponds to a normal combustion step for methane fuel. When considering extinction, reaction (GR1) is enhanced by two additional steps: $\mathrm{CH}_{4}+2 \mathrm{O}_{2} \rightarrow\left(\mathrm{CH}_{4}\right)^{*}+2 \mathrm{O}_{2}(\mathrm{GR} 2)$, and $\left(\mathrm{CH}_{4}\right)^{*}+2 \mathrm{O}_{2} \rightarrow \mathrm{CO}_{2}+2 \mathrm{H}_{2} \mathrm{O}(\mathrm{GR} 3)$, where global reaction (GR2) describes flame extinction, treated as a transformation of fresh fuel $\mathrm{CH}_{4}$ into non-burning fuel $\left(\mathrm{CH}_{4}\right)^{*}$, and global reaction (GR3) describes re-ignition, treated as a normal combustion step for $\left(\mathrm{CH}_{4}\right)^{*}$. The introduction of two separate combustion steps (GR1) and (GR3) allows for a separate treatment in the model of extinction and re-ignition phenomena. The list of transported species in the combustion model includes $\mathrm{CH}_{4}$, $\left(\mathrm{CH}_{4}\right)^{*}, \mathrm{O}_{2}, \mathrm{CO}_{2}$ and $\mathrm{H}_{2} \mathrm{O}$.

The closure expressions for the chemical reaction rates are based on the EDC model [18]:

$$
\begin{array}{r}
\overline{\dot{\omega}_{G R 1}^{\prime \prime \prime}}=(1-F E F) \times C_{E D C}\left(\frac{\bar{\rho}}{\tau_{t}}\right) \times \min \left(\tilde{Y}_{C H_{4}}, \frac{\widetilde{Y}_{O_{2}}}{r_{s}}\right) \\
\overline{\dot{\omega}_{G R 2}^{\prime \prime \prime}}=F E F \times C_{E D C}\left(\frac{\bar{\rho}}{\tau_{t}}\right) \times \min \left(\tilde{Y}_{C H_{4}}, \frac{\widetilde{Y}_{O_{2}}}{r_{s}}\right) \\
\overline{\dot{\omega}_{G R 3}^{\prime \prime \prime}}=F I F \times C_{E D C}\left(\frac{\bar{\rho}}{\tau_{t}}\right) \times \min \left(\tilde{Y}_{\left(C H_{4}\right)^{*}}, \frac{\tilde{Y}_{O_{2}}}{r_{s}}\right)
\end{array}
$$

where FEF and FIF designate the flame extinction and re-ignition factors, where $C_{E D C}$ is a model coefficient $\left(C_{E D C}=4\right), \bar{\rho}$ the (LES-filtered) mass density, $\tau_{t}$ the subgrid-scale turbulent mixing time scale $\left(\tau_{t}=\left(1 / C_{e}\right) \times\right.$ 
$\left(\Delta /\left(k_{S G S}\right)^{1 / 2}\right)$, with $C_{e}$ a dynamic model coefficient, $\Delta$ the local grid cell size, and $k_{S G S}$ the subgrid-scale TKE), where $\widetilde{Y}_{C_{4}}, \widetilde{Y}_{\left(C_{4}\right)^{*}}$ and $\widetilde{Y}_{O_{2}}$ are the (LES-filtered) mass fractions of fuel, non-burning fuel and oxygen, and $r_{s}$ is the stoichiometric oxygen-to-fuel mass ratio .

In Eq. 1, the flame extinction factor $F E F$ takes values between 0 and 1 and gives a measure of the local probability of flame extinction. Flame extinction is treated via a critical Damköhler number criterion: $F E F=0$ if $D a \geqslant D a_{c}$ (no extinction) and $F E F=1$ if $D a<D a_{c}$ (full extinction). We use $D a_{c}=1[16]$ and write:

$$
D a=C \times \frac{\left(1 / \chi_{s t}\right)}{\exp \left(T_{a} / T_{s t}\right)}
$$

where $C$ and $T_{a}$ are model parameters, $T_{s t}$ is the flame temperature and $\chi_{s t}$ the rate of fuel-air mixing. In Eq. 2, $\left(1 / \chi_{s t}\right)$ represents a mixing time scale while $(1 / C) \exp \left(T_{a} / T_{s t}\right)$ represents a chemical time scale; $T_{a}$ is an effective activation temperature which represents the sensitivity of the combustion chemistry to changes in temperature. $C$ and $T_{a}$ are fuel dependent properties that have been calibrated in the present study through comparison with direct numerical simulation data on extinction limits of methane-air laminar diffusion flames [24], $C=1.9616 \times 10^{10} \mathrm{~s}^{-1}$ and $T_{a}=36856 \mathrm{~K}$. The flamebased quantities $T_{s t}$ and $\chi_{s t}$ are obtained via subgrid-scale models and are discussed next.

The flame temperature $T_{s t}$ is described as a weighted average between the adiabatic flame temperature $T_{s t}^{a d}$ and the mixing temperature $T_{s t}^{m}$, using the deficit enthalpy variable $H_{s t}$ as a weight coefficient: 


$$
T_{s t}=\left(1+H_{s t}\right) T_{s t}^{a d}-H_{s t} T_{s t}^{m}
$$

$T_{s t}^{a d}$ is the temperature that would be obtained with adiabatic combustion (i.e., without radiation losses, convective wall losses, or an evaporating spray); we use $T_{s t}^{a d}=2240 \mathrm{~K} . T_{s t}^{m}$ is the temperature that would be obtained with pure fuel-air mixing and without combustion; we use $T_{s t}^{m}=293 \mathrm{~K}$. The deficit enthalpy variable $H_{s t}$ takes values between $(-1)$ and 0 and gives a measure of the magnitude of non-adiabatic effects at the flame location: $H_{s t}=0$ under adiabatic burning conditions while $H_{s t}=-1$ under extinguished conditions. We write:

$$
H_{s t}=-\frac{\left(\widetilde{h}_{s}^{a d}-\widetilde{h}_{s}\right)}{\left(\widetilde{h}_{s}^{a d}-\widetilde{h}_{s}^{m}\right)}
$$

where $\widetilde{h}_{s}, \widetilde{h}_{s}^{a d}$ and $\widetilde{h}_{s}^{m}$ are the (LES-filtered) sensible enthalpy, adiabatic sensible enthalpy and mixing sensible enthalpy, respectively. $\widetilde{h}_{s}$ is a principal variable in the FireFOAM system of governing equations and is therefore obtained directly from the original FireFOAM transport equation for energy. $\widetilde{h}_{s}^{a d}$ and $\widetilde{h}_{s}^{m}$ are obtained from additional modified transport equations. The calculation of $\widetilde{h}_{s}^{a d}$ is identical to that of $\widetilde{h}_{s}$ except that the thermal radiation and spray evaporation terms in the enthalpy equation are set to 0 and boundary conditions are changed to adiabatic. Similarly, the calculation of $\widetilde{h}_{s}^{m}$ is identical to that of $\widetilde{h}_{s}^{a d}$ except that the combustion term in the enthalpy equation is set to 0 . This treatment provides a simple method to account for local decreases in flame temperature due to the presence of thermal losses. In the present study, thermal losses are due to both emission of radiant energy and cooling from the evaporating water mist. 
Furthermore, the rate of fuel-air mixing $\chi_{s t}$ in Eq. 2 is described based on a classical closure expression for the LES-filtered scalar dissipation rate, $\widetilde{\chi}=$ $2\left(D+D_{t}\right)|\nabla \widetilde{Z}|^{2}$, where $D$ and $D_{t}$ are the molecular and turbulent thermal diffusivities, and $\widetilde{Z}$ is the mixture fraction. Replacing mixture fraction by its expression in terms of fuel and oxygen mass, we get:

$$
\chi_{s t}=2\left(D+D_{t}\right)\left(\frac{r_{s} \nabla \tilde{Y}_{C H_{4}}-\nabla \tilde{Y}_{O_{2}}}{r_{s}+Y_{O_{2}, a i r}}\right)^{2}
$$

where $Y_{O_{2} \text {,air }}$ is the oxygen mass fraction in air, $Y_{O_{2}, \text { air }}=0.233$.

Finally, in Eq. 1, the flame re-ignition factor FIF takes values between 0 and 1 and gives a measure of the local probability of flame re-ignition. Flame re-ignition is treated via a critical gas temperature criterion: $F I F=0$ if $\widetilde{T} \leqslant T_{i g n}$ (no re-ignition) and $F I F=1$ if $\widetilde{T} \geqslant T_{i g n}$ (re-ignition). We use $T_{i g n}=1100 \mathrm{~K}[25]$.

\subsection{Resolution and computational cost}

The computational domain is 200 -cm-wide in the cross-stream $x$-direction, 85-cm-deep in the spanwise $y$-direction and 200-cm-high in the vertical $z$ direction. The computational grid is generated using the OpenFOAM mesh tool called SnappyHexMesh [14]. The grid is block-structured and features three levels of grid resolution (Fig. 1). The level-one refinement block contains the flame region and features a uniform grid with cubic cells of size equal to $4.167 \mathrm{~mm}$ (i.e., 12 grid cells across the burner width). The level-one refinement block is 40-cm-wide, 60-cm-deep and 60-cm-high. The level-two refinement block features a uniform grid with cubic cells of size equal to 8.333 $\mathrm{mm}$; this block is 60 -cm-wide, 80 -cm-deep and 80 -cm-high. The level-three 


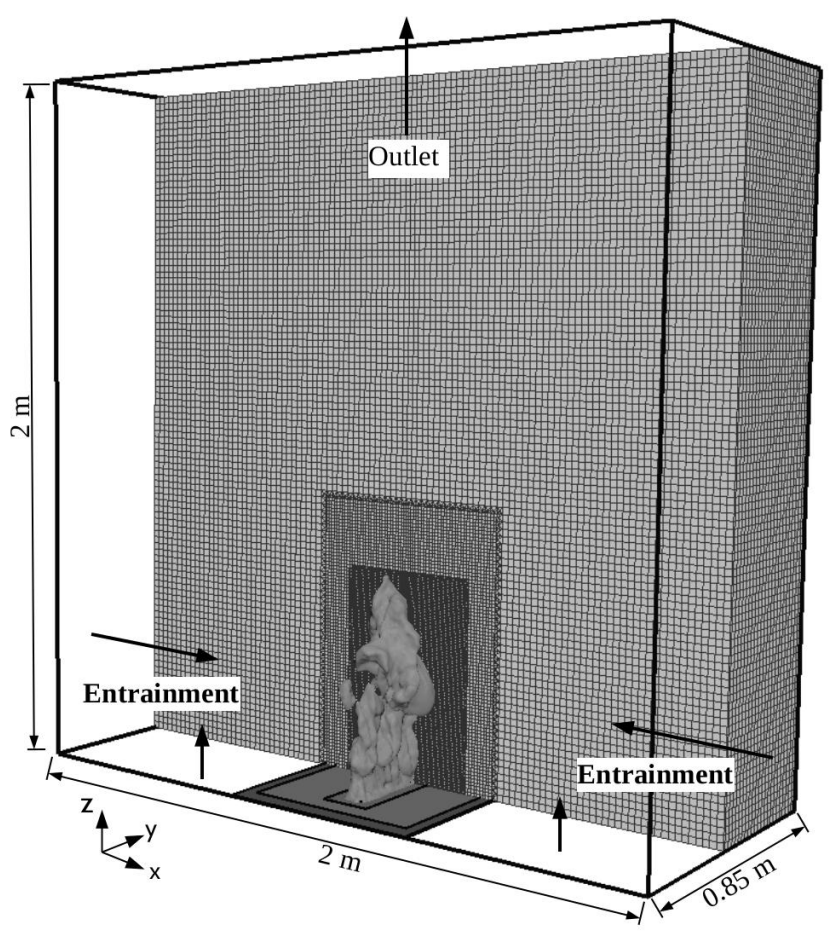

Figure 1: Computational domain and multi-level mesh refinement; the Wolfhard-Parker burner is $5 \mathrm{~cm}$ wide in the $x$-direction and $50 \mathrm{~cm}$ deep in the $y$-direction; the flame height is approximately $50 \mathrm{~cm}$.

refinement block contains the far-field air entrainment and plume regions and features a uniform grid with cubic cells of size equal to $16.67 \mathrm{~mm}$. The total number of cells is 1.85 million.

Previous work corresponding to simulations of similar flames but without mist included a grid-convergence study and concluded that for the present moderate-Reynolds-number flames, a $4 \mathrm{~mm}$ grid resolution is sufficiently fine to achieve approximate grid independence of the LES solutions [23]. The present LES simulations are well-resolved in the sense that anywhere in the 
flow, the local ratio of mean grid-resolved TKE divided by mean total TKE (equal to the sum of mean grid-resolved TKE plus mean subgrid-scale TKE) is above $90 \%$. In addition, the ratio of mean subgrid-scale eddy viscosity divided by molecular viscosity remains less than 1 .

The accuracy of the Lagrangian-based treatment of the water mist is controlled by the number of numerical droplets $n_{i n j}$ injected into the computational domain per second. To minimize sampling error, we use $n_{i n j}=100,000$ [26].

All simulations are $14 \mathrm{~s}$ in duration. Turbulent statistics are collected for a duration of at least $10 \mathrm{~s}$ at the end of each simulation, once the flow and flame become statistically stationary. Each simulation is run using 40 processors on a large-scale Linux cluster; with typical runs requiring on the order of 1500 hours of CPU time.

\section{Results and discussion}

\subsection{Mist-flow interactions}

We first consider a series of preliminary test simulations in which the extinction model is disabled (the flame power remains equal to $50 \mathrm{~kW}$ ). These test simulations provide insight into the interactions between the water mist and the air co-flow. We consider here our baseline configuration corresponding to a mono-disperse spray of $30 \mu \mathrm{m}$ droplets moving with the air co-flow at $29 \mathrm{~cm} / \mathrm{s}$. This baseline configuration does not include a protective co-flow.

While the water droplets are initially injected at the air co-flow velocity, the droplets are observed to quickly decelerate due to gravity, causing in turn a deceleration of the air flow due to drag. Thus, the momentum exchange 
between the gas and liquid phases is capable of changing the air entrainment process into the flame zone. The intensity of the momentum exchange between gas and liquid depends on the water load. For small liquid-to-gas mass flow rate ratios (e.g., of order 1\% or smaller), the droplets do not disturb the air co-flow significantly and the buoyant motions are strong enough to successfully entrain the droplets into the entire length of the flame zone (Fig. 2-a). In contrast, for large liquid-to-gas mass flow rate ratios (e.g., larger than 10\%), the droplets significantly modify the air co-flow and the buoyant motions are now too weak to entrain the droplets beyond a small region at the base of the flame (Fig. 2-b). Thus, different water loads will result in different distributions of liquid and vaporized water along the flame, irrespective of how the flame responds.

(a)
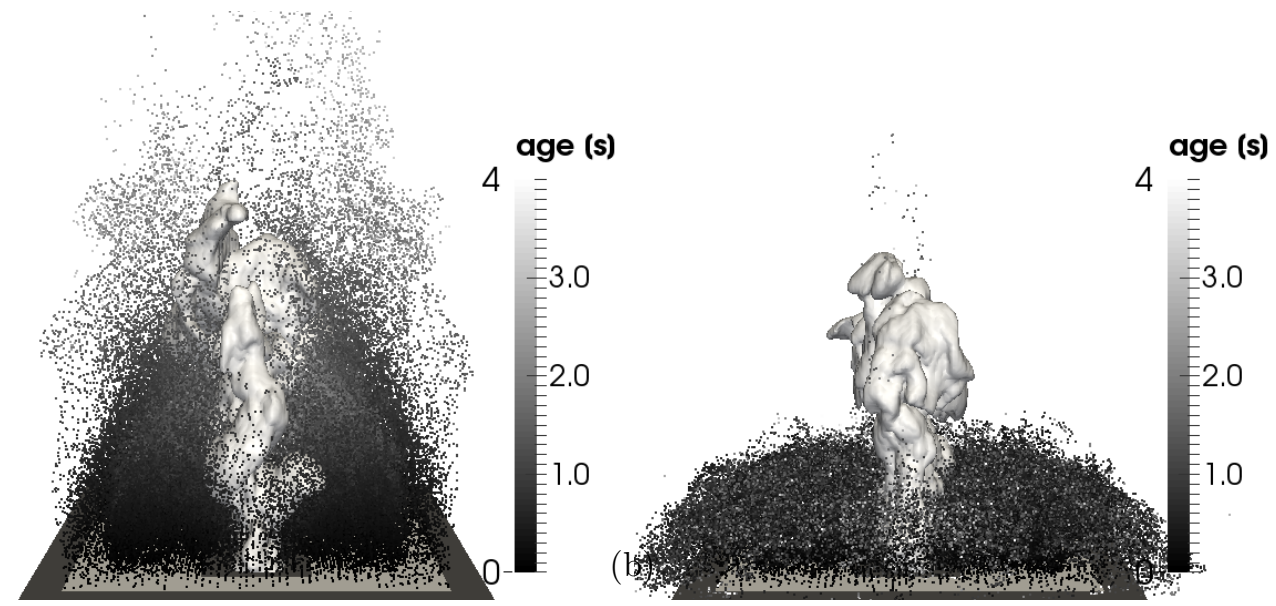

Figure 2: Instantaneous mist droplet distribution in test simulations without flame extinction; the air co-flow mass flow rate is $100 \mathrm{~g} / \mathrm{s}$; the mist corresponds to $30 \mu \mathrm{m}$ droplets with different mist mass flow rates: a) $\dot{m}_{w}=2 \mathrm{~g} / \mathrm{s}$; b) $\dot{m}_{w}=18 \mathrm{~g} / \mathrm{s}$. In the pictures, the flame is shown as an iso-contour of the heat release rate per unit volume $\left(200 \mathrm{~kW} / \mathrm{m}^{3}\right)$. 


\subsection{Mist-flame interactions}

We now turn to a discussion of simulations in which the extinction model is activated. We consider three configurations: (1) the baseline configuration, called Case 1, discussed in the previous section (monodisperse spray with droplet diameter equal to $30 \mu \mathrm{m}$, variable mist mass flow rate $\dot{m}_{w}$ between 0 and $18 \mathrm{~g} / \mathrm{s}$, and without a protective co-flow); (2) a similar configuration, called Case 2, with $60 \mu \mathrm{m}$ droplets; and (3) a modified configuration, called Case 3, with a protective co-flow (monodisperse spray with droplet diameter equal to $30 \mu \mathrm{m}$, mist mass flow rate $\dot{m}_{w}$ equal to $18 \mathrm{~g} / \mathrm{s}$ ). In Case 3 , we consider four values of the protective co-flow velocity: $w_{a i r, p}=20,30,40$ and $50 \mathrm{~cm} / \mathrm{s}$; higher flow velocities provide higher levels protection of the flame from the water mist.

Figure 3 presents the combustion efficiency $\chi_{a}$ as a function of the performance indicator $I_{\text {global }}$ discussed in the Introduction section. $\chi_{a}$ is defined as the ratio of the (time- and volume-averaged) heat release rate (HRR) of the flame with mist divided by HRR of the flame without mist (i.e., $50 \mathrm{~kW}$ ). $I_{\text {global }}$ is the theoretical evaporative cooling power (ECP) of the mist, $\left(\dot{m}_{w} \Delta H_{v}\right)$, divided by HRR of the flame without mist; we use $\Delta H_{v}=3.7 \mathrm{MJ} / \mathrm{kg}$.

Figure 3 shows that consistent with previous results found in the literature (e.g., [9]), the response of the flame in Cases 1 and 2 corresponds to a decrease in flame strength with increasing values of $I_{\text {global }}$. The simulated flames correspond to values of $I_{\text {global }}$ between 0 and 1.33: in Case 1, total flame extinction is (almost) achieved for $I_{\text {global }} \approx 0.8$, with a small residual flame remaining near the fuel injection plane. In contrast, in Case 2, even at the highest simulated water mist load, the flame remains partially burning. The 
Case 2 flame requires higher values of $I_{\text {global }}$ for total extinction. In other words, Fig. 3 suggests that the mist with $30 \mu \mathrm{m}$ droplets is more efficient than the mist with $60 \mu \mathrm{m}$ droplets (total extinction is achieved for smaller amounts of injected liquid water).

Case 3 corresponds to a dramatically different flame response. Case 3 includes four simulations corresponding to different values of $w_{a i r, p}$. All simulations feature the same value of the mist mass flow rate $\dot{m}_{w}$ and therefore appear on a vertical line in Figure $3, I_{\text {global }}=1.33$. At high values of $w_{a i r, p}$, the flame is insulated from the outer co-flow and has limited interaction with the mist; the combustion efficiency is high. At low values of $w_{a i r, p}$, the flame interacts strongly with the outer co-flow; the combustion efficiency is low. These results illustrate the failure of $I_{\text {global }}$ to give a robust and meaningful measure of the spray effectiveness: the same value $I_{\text {global }}=1.33$ corresponds to flames with different levels of combustion efficiency, $\chi_{a} \approx 10 \%, 20 \%, 50 \%, 80 \%$ for $w_{a i r, p}=20,30,40,50 \mathrm{~cm} / \mathrm{s}$, respectively.

As mentioned in the introduction, the main deficiency in the performance indicator $I_{\text {global }}$ is that it uses the total amount of liquid water delivered rather than the fraction that evaporates and interacts with the flame. We consider now a modified version of the indicator, noted $I_{\text {global }}^{*}$, based on the simulated (time- and volume-averaged) evaporation rate of the liquid phase: $I_{\text {global }}^{*}=\left(\dot{\omega}_{w} \Delta H_{v}\right) / \dot{Q}_{c}$, where $\dot{\omega}_{w}$ is the global mass evaporation rate of liquid water (in units of $\mathrm{kg} / \mathrm{s}$ ),

Figure 4 presents the combustion efficiency $\chi_{a}$ as a function of the modified performance indicator $I_{\text {global }}^{*}$. It is seen that the values of $I_{\text {global }}^{*}$ range between 0 and 0.4 and as expected, are much lower than those of $I_{\text {global }}$ 


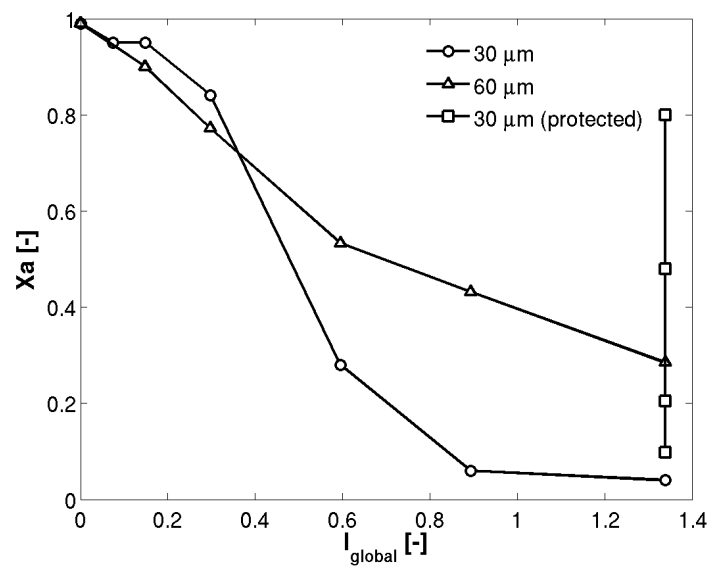

Figure 3: Combustion efficiency $\chi_{a}$ as a function of $I_{\text {global }}$ for Case 1 (circles), Case 2 (triangles) and Case 3 (squares). Case 1 and Case 2 data points correspond to different values of the mist mass flow rate $\dot{m}_{w}$; Case 3 data points correspond to different values of the protective co-flow velocity $w_{a i r, p}$ (from high to low velocity as one moves from the top to bottom points in the plot).

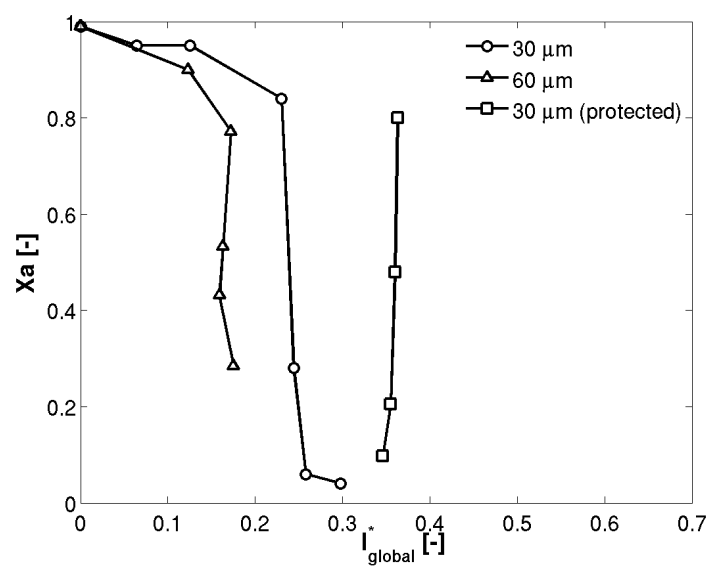

Figure 4: Combustion efficiency $\chi_{a}$ as a function of $I_{\text {global }}^{*}$. See Fig. 3. 
$\left(\dot{\omega}_{w}<\dot{m}_{w}\right)$. The curves in Fig. 4 feature an abrupt transition from high to low values of the combustion efficiency: this transition occurs at $I_{\text {global }}^{*} \approx 0.25$, 0.15 and 0.35 , in Cases 1, 2 and 3, respectively.

When comparing Case 1 and Case 2, it is seen that Case 2 corresponds to less evaporation ( $I_{\text {global }}^{*}$ takes lower values) and a more efficient spray (transition to extinction occurs at a lower critical value of $I_{\text {global }}^{*}$ ). In other words, and in contrast to the conclusion drawn earlier from Fig. 3, Fig. 4 suggests that the mist with $60 \mu \mathrm{m}$ droplets is more efficient than the mist with $30 \mu \mathrm{m}$ droplets (total extinction is achieved for smaller amounts of evaporated liquid water). Thus, while the Case 2 mist is less efficient than the Case 1 mist at delivering water to the flame (Fig. 3), the delivered water in Case 2 is more efficient than that in Case 1 at suppressing the flame (Fig. 4).

When comparing Case 1 and Case 3, it is seen that Case 3 corresponds to more evaporation ( $I_{\text {global }}^{*}$ takes higher values) and a less efficient spray (transition to extinction occurs at a higher critical value of $I_{\text {global }}^{*}$ ). The result that Case 3 corresponds to more evaporation is somewhat surprising since it may have been assumed that the protection provided by the inner co-flow would negatively impact evaporation. This result is explained next by considering the spatial distribution of HRR and ECP as a function of elevation above the burner.

We now consider spatially-resolved information and compare combustion and evaporation intensities inside the flame region. This comparison is made in the context of reference simulations in which the extinction model is disabled. The idea here is to evaluate the spray dispersion and evaporation properties prior to suppression and to thereby differentiate between mist- 
flame configurations in which the bulk of the evaporation takes place inside the flame and mist-flame configurations in which the bulk of the evaporation takes place downstream. Figure 5 presents the vertical variations of horizontally-integrated HRR and ECP, $\dot{Q}_{c}^{\prime}$ and $\left(\dot{\omega}_{w}^{\prime} \Delta H_{v}\right)$ (both in units of $\mathrm{W} / \mathrm{m})$. Figure 5 includes results from three simulations with similar values of the combustion efficiency: a Case 1 simulation with $\dot{m}_{w}=8 \mathrm{~g} / \mathrm{s}$ and $\chi_{a} \approx 0.28$; a Case 2 simulation with $\dot{m}_{w}=18 \mathrm{~g} / \mathrm{s}$ and $\chi_{a} \approx 0.28$; and a Case 3 simulation with $\dot{m}_{w}=18 \mathrm{~g} / \mathrm{s}, w_{a i r, p}=30 \mathrm{~cm} / \mathrm{s}$ and $\chi_{a} \approx 0.21$.

Figure 5 shows that the HRR profiles are similar in all three cases (the flame power is equal to $50 \mathrm{~kW}$ ) with some variations associated with the impact of the spray and/or protective co-flow on fuel-air mixing. Figure 5 also shows that the ECP profiles feature more pronounced variations. Case 1 and Case 3 feature high evaporation rates up to mid-flame height, $z \leq 25 \mathrm{~cm}$, and negligible evaporation beyond. Case 2 features high evaporation rates at the base of the flame, $z \leq 10 \mathrm{~cm}$, and negligible evaporation at higher elevations. This result for Case 2 is consistent with the discussion of mist-flow interactions in the previous section that suggests that high concentrations of small droplets (Fig. 2-b) or equivalently more moderate concentrations of larger droplets can limit the region of influence of the spray to the base of the flame.

Figure 5 sheds some light on some of the results presented in Fig. 4. The total rate of evaporation $\dot{\omega}_{w}$ is equal to the area under the ECP curves plotted in the figure and it is seen that Case 1 evaporates more than Case 2 but evaporates less than Case 3. In Case 3, increasing values of $w_{a i r, p}$ result in more evaporation occurring in the downstream portion of the flame as well 

of the flame.

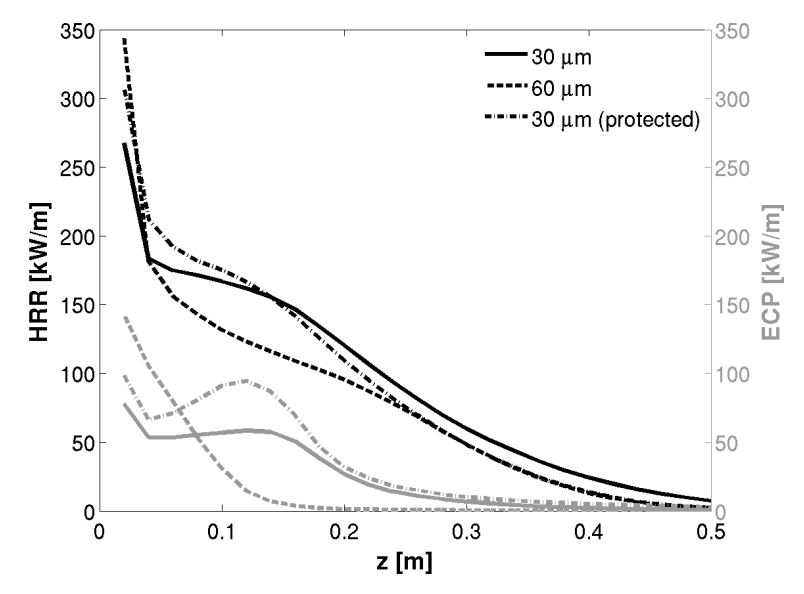

Figure 5: Heat release rate per unit height (black) and evaporative cooling power per unit height (grey) as a function of elevation for three representative simulations corresponding to Cases 1, 2 and 3. Reference simulations without flame extinction. The flame height is approximately $50 \mathrm{~cm}$.

as in the plume region; this result explains the surprisingly high values of $\dot{\omega}_{w}$ found in Case 3. Figure 5 also shows that the ECP curves take comparable values near the base of the flame (at $z \leq z_{\text {base }}$ with $z_{\text {base }} \approx 10 \mathrm{~cm}$ ) but differ significantly at higher elevations. Because the three cases presented in the figure correspond to similar values of the combustion efficiency, one can then postulate that $\chi_{a}$ is controlled by the rate of evaporation in the base region

as the ratio of mean ECP integrated over the flame base region divided by mean HRR integrated over the same region: 


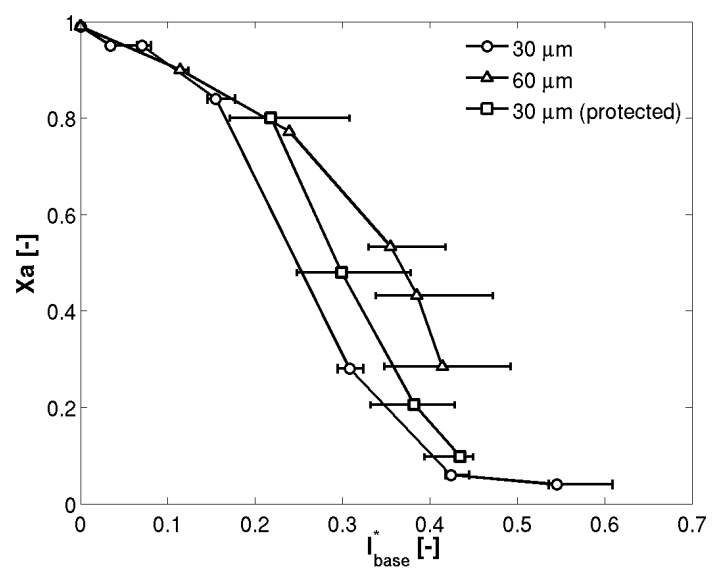

Figure 6: Combustion efficiency $\chi_{a}$ as a function of $I_{b a s e}^{*}$. See Fig. 3.

$$
I_{\text {base }}^{*}=\frac{\left(\int_{0}^{z_{\text {base }}}\left(\dot{\omega}_{w}^{\prime} \Delta H_{v}\right) d z\right)}{\left(\int_{0}^{z_{\text {base }}} \dot{Q}_{c}^{\prime} d z\right)}
$$

Our baseline choice for the extent of the flame base region is $\left(z_{\text {base }} / L_{f}\right)=$ 0.2 , where $L_{f}$ is the flame height $\left(L_{f} \approx 50 \mathrm{~cm}\right)$, or $z_{\text {base }}=10 \mathrm{~cm}$. Figure 6 presents the combustion efficiency $\chi_{a}$ as a function of $I_{\text {base }}^{*}$. The horizontal bars in the figure correspond to variations in the estimates of $I_{\text {base }}^{*}$ when $\left(z_{\text {base }} / L_{f}\right)$ changes from 0.1 to 0.3 . While there is some scatter, it is seen that the collapse of the data is much better than that obtained in Figs. 3 and 4 .

This result supports the idea that flame suppression is controlled by the ability of the spray to weaken the flame in the base region. The importance of the base region comes from its role as a high combustion intensity region (HRR is maximum at low elevations) as well as its role for flame stabilization (flame elements at low elevations have a large impact on HRR at downstream 
locations).

\section{Conclusion}

This paper presents a numerical study of suppression performance by a water mist injected at the base of a buoyant, turbulent, methane-fueled, slot burner diffusion flame with an emphasis on engineering- and research-level indicators used to evaluate spray effectiveness. The study is based on a series of well-resolved large eddy simulations performed with FireFOAM [12] and using a modified version recently enhanced with a new flame extinction model $[15,23]$.

The present study provides basic information on the flame response to changes in the water mist load and in particular on the variations of the combustion efficiency with respect to droplet size, number density or the presence of a protective co-flow. Results highlight the limitations of global spray effectiveness indicators that are based on delivered water rather than evaporated water, or are based on evaporated water without an appreciation for where the evaporation is taking place with respect to the flame structure. Results also show that a local spray effectiveness indicator defined as the mean evaporative cooling power of the mist, measured in the flame base region, divided by the mean heat release rate of the flame, also measured in the flame base region, provides a fair collapse of the flame extinction data.

\section{Acknowledgments}

This project is supported by the U.S. National Science Foundation (GOALI Award \# 1236788) and by FM Global. The authors would like to gratefully 
acknowledge helpful discussions with Drs. Yi Wang and Karl Meredith from

FM Global. The authors acknowledge the University of Maryland supercomputing resources (http://www.it.umd.edu/hpcc) made available in conducting the research reported in this paper. This work also used the Extreme Science and Engineering Discovery Environment (XSEDE), which is supported by National Science Foundation grant number TG-CTS140046.

\section{References}

[1] D. J. Rasbash, Fire Safety Science, Proc. First International Symposium, International Association for Fire Safety Science (1986) 1145-1163.

[2] G. Grant, J. Brenton, D. Drysdale, Prog. Energy Combust. Sci. 26 (2000) 79-130.

[3] R. Friedman, Fire Protection Handbook, National Fire Protection Association (19th Ed.) (2003) 2/83-2/96.

[4] A. M. Lentati, H. K. Chelliah, Combust. Flame 115 (1998) 158-179.

[5] A. M. Lentati, H. K. Chelliah, Proc. Combust. Inst. 27 (1998) 2839 2846.

[6] A. K. Lazzarini, R. H. Krauss, H. K. Chelliah, G. T. Linteris, Proc. Combust. Inst. 28 (2000) 2939-2945.

[7] P. G. Arias, H. G. Im, P. Narayanan, A. Trouvé, Proc. Combust. Inst. 33 (2011) 2591-2597.

[8] R. Ananth, R. C. Mowrey, Combust. Sci. Tech. 180 (2008) 1659-1692. 
[9] K. Prasad, C. Li, K. Kailasanath, C. Ndubizu, R. Ananth, P. A. Tatem, Combust. Sci. Tech. 132 (1998) 325-364.

[10] K. Prasad, C. Li, K. Kailasanath, Fire Safety J. 33 (1999) 185-212.

[11] J. P. White, E. D. Link, A. C. Trouvé, P. B. Sunderland, A. W. Marshall, J. A. Sheffel, M. L. Corn, M. B. Colket, M. Chaos, H.-Z. Yu, Fire Safety J. 76 (2015) 74-84.

[12] FM Global, FireFOAM, available at: <https://github.com/ fireFoam-dev>, accessed: 11-30-2015.

[13] Y. Wang, K. V. Meredith, X. Zhou, P. Chatterjee, Y. Xin, M. Chaos, N. Ren, S. B. Dorofeev, Fire Safety Science, Proc. Eleventh International Symposium, International Association for Fire Safety Science (2014) 1170-1183.

[14] OpenCFD Ltd., OpenFOAM, available at: <http://www.openfoam. com>, accessed: 11-30-2015.

[15] S. Vilfayeau, N. Ren, Y. Wang, A. Trouvé, Proc. Combust. Inst. 35 (2015) 2563-2571.

[16] V. Lecoustre, P. Narayanan, H. R. Baum, A. Trouvé, Fire Safety Science, Proc. Tenth International Symposium, International Association for Fire Safety Science (2011) 583-595.

[17] C. Fureby, G. Tabor, H. G. Weller, A. D. Gosman, Phys. Fluids 9 (1997) 1416-1429. 
[18] B. Magnussen, B. H. Hjertager, Proc. Combust. Inst. 16 (1976) 719-729.

[19] J. C. Chai, P. Rath, Intl. Workshop on Discrete-Ordinates and FiniteVolume Methods for Radiation Heat Transfer (2006).

[20] S. Subramaniam, Prog. Energy Combust. Sci. 39 (2013) 215-245.

[21] N. Nordin, Complex chemistry modeling of diesel spray combustion, PhD thesis, Chalmers University of Technology, 2001.

[22] G. B. Macpherson, N. Nordin, H. G. Weller, Commun. Numer. Meth. Engng 25 (2009) 263-273.

[23] S. Vilfayeau, J. White, P. B. Sunderland, A. W. Marshall, A. Trouvé, Fire Safety J. (2015). Submitted for publication.

[24] S. H. Chan, J. Q. Yin, B. J. Shi, Combust. Flame 112 (1998) 445-456.

[25] J. C. Hewson, A. R. Kerstein, Combust. Sci.Technol. 174 (2002) 35-66.

[26] T. Myers, A. W. Marshall, Fire Safety J. (2015). Submitted for publication. 
502

\section{Figure Captions}

Figure 1. Computational domain and multi-level mesh refinement; the WolfhardParker burner is $5 \mathrm{~cm}$ wide in the $x$-direction and $50 \mathrm{~cm}$ deep in the $y$ direction; the flame height is approximately $50 \mathrm{~cm}$.

Figure 2. Instantaneous mist droplet distribution in test simulations without flame extinction; the air co-flow mass flow rate is $100 \mathrm{~g} / \mathrm{s}$; the mist corresponds to $30 \mu \mathrm{m}$ droplets with different mist mass flow rates: a) $\dot{m}_{w}=2 \mathrm{~g} / \mathrm{s}$; b) $\dot{m}_{w}=18 \mathrm{~g} / \mathrm{s}$. In the pictures, the flame is shown as an iso-contour of the heat release rate per unit volume $\left(200 \mathrm{~kW} / \mathrm{m}^{3}\right)$.

Figure 3. Combustion efficiency $\chi_{a}$ as a function of $I_{g l o b a l}$ for Case 1 (circles), Case 2 (triangles) and Case 3 (squares). Case 1 and Case 2 data points correspond to different values of the mist mass flow rate $\dot{m}_{w}$; Case 3 data points correspond to different values of the protective co-flow velocity $w_{a i r, p}$ (from high to low velocity as one moves from the top to bottom points in the plot).

Figure 4. Combustion efficiency $\chi_{a}$ as a function of $I_{\text {global }}^{*}$. See Fig. 3.

Figure 5. Heat release rate per unit height (black) and evaporative cooling power per unit height (grey) as a function of elevation for three representative simulations corresponding to Cases 1,2 and 3. Reference simulations without 
${ }_{521}$ flame extinction. The flame height is approximately $50 \mathrm{~cm}$.

${ }_{522}$ Figure 6. Combustion efficiency $\chi_{a}$ as a function of $I_{b a s e}^{*}$. See Fig. 3. 